\title{
Adiabatic energization in the ring current and its relation to other source and loss terms
}

\author{
M. W. Liemohn, J. U. Kozyra, and C. R. Clauer \\ Space Physics Research Laboratory, University of Michigan, Ann Arbor, Michigan, USA
}

G. V. Khazanov

National Space Science and Technology Center, NASA Marshall Space Flight Center, Huntsville, Alabama, USA

M. F. Thomsen

Atmospheric and Space Sciences, Los Alamos National Laboratory, Los Alamos, New Mexico, USA

Received 1 August 2001; revised 13 September 2001; accepted 21 September 2001; published 24 April 2002.

[1] The influence of adiabatic energization and deenergization effects, caused by particle drift in radial distance, on ring current growth rates and loss lifetimes is investigated. Growth and loss rates from simulation results of four storms (5 June 1991, 15 May 1997, 19 October 1998, and 25 September 1998) are examined and compared against the $y$ component of the solar wind electric field $\left(E_{\gamma, \mathrm{sw}}\right)$. Energy change rates with and without the inclusion of adiabatic energy changes are considered to isolate the influence of this mechanism in governing changes of ring current strength. It is found that the influence of adiabatic drift effects on the energy change rates is very large when energization and deenergization are considered separately as gain and loss mechanisms, often about an order of magnitude larger than all other source or loss terms combined. This is true not only during storm times, when the open drift path configuration of the hot ions dominates the physics of the ring current, but also during quiet times, when the small oscillation in $L$ of the closed trajectories creates a large source and loss of energy each drift orbit. However, the net energy change from adiabatic drift is often smaller than other source and loss processes, especially during quiet times. Energization from adiabatic drift dominates ring current growth only during portions of the main phase of storms. Furthermore, the net-adiabatic energization is often positive, because some particles are lost in the inner magnetosphere before they can adiabatically deenergize. It is shown that the inclusion of only this net-adiabatic drift effect in the total source rate or loss lifetime (depending on the sign of the net-adiabatic energization) best matches the observed source and loss values from empirical Dst predictor methods (that is, for consistency, these values should be compared between the calculation methods). While adiabatic deenergization dominates the loss timescales for all $E_{y, \mathrm{sw}}$ values, there is a characteristic decrease in the loss lifetime for higher $E_{y, \mathrm{sw}}$, seen computationally and observationally. It is shown that these shorter loss lifetimes at higher $E_{y, \mathrm{sw}}$ are caused primarily by enhanced dayside flow out. The simulation results show that, on average, flow out is a larger energy loss term than charge exchange (the next biggest loss term for the ring current) for all $E_{y, \mathrm{sw}}$ values. INDEX TERMS: 2778 Magnetospheric Physics: Ring current; 2788 Magnetospheric Physics: Storms and substorms; 2753 Magnetospheric Physics: Numerical modeling; 2730 Magnetospheric Physics: Magnetosphere - inner

\section{Introduction}

[2] The ring current is not symmetric during the main phase of geomagnetic storms but, rather, is highly asymmetric in local time with peak intensity near dusk/midnight. This has been shown both observationally [e.g., Greenspan and Hamilton, 2000; Jorgensen et al., 2001; Burch et al., 2001; Pollock et al., 2001; Mitchell et al., 2001; Reeves and Spence, 2001] as well as computationally [e.g., Kozyra et al., 1998; Liemohn et al., 1999, 2001; Jordanova et al., 1999; Ebihara and Ejiri, 2000; Kozyra et al., 2002]. Because of this, adiabatic energy gain and loss due to radial motion in the inner magnetosphere (that is, drift-induced changes in $L$ shell) should not be considered a negligible term in the energy balance of the ring current. This

Copyright 2002 by the American Geophysical Union. 0148-0227/02/2001JA000243 factor was previously considered only as part of the rapid source term [e.g., Lyons and Williams, 1980; Lee et al., 1983] but was thought to be insignificant as a loss process because it was believed the particles were rapidly trapped into Earth-orbiting trajectories. Any cross- $L$ drift of the captured population was thought to average to zero. However, because most of the hot ions (energies greater than about $1 \mathrm{keV}$, the major carriers of the energy and thus the current) are on open drift paths [e.g., Chen et al., 1994], making only one pass through the inner magnetosphere, this term can play a large role in the total strength of the ring current. All of this has implications for the magnitude and time variability of $D s t^{*}$, the component of the average groundbased magnetic perturbation from near-Earth current systems (mainly the ring current [e.g., Gonzalez et al., 1994]).

[3] In this report, the significance of the adiabatic energy gain and loss due to radial drift is considered by examining results from the ion transport model of Liemohn et al. [2001] and Kozyra et al. 
[2002]. These results are then compared with the solar wind dependencies of various $D s t^{*}$ predictor methods.

\section{Approach}

[4] This study uses results from a kinetic ring current model that solves the gyration and bounce-averaged Boltzmann equation inside of geosynchronous orbit [Fok et al., 1993; Jordanova et al., 1996; Liemohn et al., 2001],

$$
\begin{aligned}
\frac{\partial f^{*}}{\partial t}+ & \frac{\partial}{\partial \vec{R}_{\perp}}\left\{\left\langle\frac{d \vec{R}_{\perp}}{d t \vec{E}}\right\rangle f^{*}\right\}+\frac{\partial}{\partial E}\left\{\left\langle\frac{d E}{d t \vec{E}}\right\rangle f^{*}\right\} \\
& +\frac{\partial}{\partial \mu_{0}}\left\{\left\langle\frac{d \mu_{0}}{d t \vec{E}}\right\rangle f^{*}\right\}=\frac{\partial}{\partial E}\left\{\left\langle\frac{d E}{d t_{\mathrm{CC}}}\right\rangle f^{*}\right\} \\
& +\frac{\partial}{\partial \mu_{0}}\left\{\left\langle D_{\mathrm{CC}}\right\rangle \frac{\partial f^{*}}{\partial \mu_{0}}\right\}-\frac{f^{*}}{\tau_{\mathrm{CE}}}-\frac{H\left(\mu_{0}-\mu_{0}, \mathrm{LC}\right) f^{*}}{0.5 \tau_{b}}
\end{aligned}
$$

for the hot-ion distribution function $f$ of a given ring current species ( $f$ is related to $f^{*}$ by a conversion to conservative variables, $f^{*}=f R_{0}^{2} \mu_{0} h\left(\mu_{0}\right) \sqrt{E}$ ). The independent variables in (1) are time $t$, geocentric position in the magnetic equatorial plane $\mathbf{R}_{\perp}$ $\left(R_{0}, \varphi\right)$, kinetic energy $E$, and the cosine of the equatorial pitch angle $\mu_{0}\left(=\cos \alpha_{0}\right)$. On the right-hand side of (1), energy decay and pitch angle scattering due to Coulomb collisions with the thermal electrons and ions in the magnetosphere stochastically rearrange the velocity space distribution, while charge exchange and atmospheric loss attenuate it according to the timescales for these processes. The Heaviside function in the final term limits its application to the loss cone. Multiple species calculations (as are usually performed) are, at present, linear additions of the results from separate runs for each particle type.

[5] Using second-order accurate numerical schemes (see Fok et al. [1993] and Jordanova et al. [1996] for a complete description of the numerical scheme), the hot-ion phase-space distribution is determined, yielding a detailed description of the ring current ion population throughout near-Earth space. The numerical grid is composed of about 500 spatial cells $\left(1.75<R_{0}<6.75 \mathrm{R}_{\mathrm{E}}, 0<\varphi\right.$ $<2 \pi)$ and roughly 3000 velocity space cells $(10 \mathrm{eV}<E<400 \mathrm{keV}$, $0<\mu_{0}<1$ ), so $f$ is calculated at 1.5 million phase space points every time step (typically $20 \mathrm{~s}$ ). The plasma source is through the outer simulation boundary, specified by measured ion fluxes from the magnetospheric plasma analyzer (MPA) [Bame et al., 1993] and the synchronous orbit particle analyzer [Belian et al., 1992], each flown on several satellites at geosynchronous orbit. The energy range of these two instruments covers that of the simulation. For the calculations presented in section 3, the geomagnetic field is assumed to be a static dipole and the convection electric field is specified by a modified McIlwain [1986] description driven by the cross-polar cap potential difference $\Delta \Phi_{\mathrm{PC}}$, with the shielding parameter specified by the midnight auroral boundary index produced by the Air Force Research Laboratory. See Liemohn et al. [2001] for further details of the model configuration and solution technique. Using solar wind data, solar and geomagnetic activity indices, and geosynchronous orbit plasma data, quantitative results are found for specific magnetic storm events.

[6] Substorm injections are not explicitly included, but changes in $\Delta \Phi_{\mathrm{PC}}$ and nightside geosynchronous ion flux as a result of substorm-related activity are both part of the calculations [e.g., DeForest and McIlwain, 1971; Chen et al., 1993; Birn et al., 1997]. Certainly both elements, numerous substorms and sustained enhanced convection, are required for the development of a large ring current [Gonzalez et al., 1994]. In the results presented in section 3, substorm effects are assumed to be included in the simulation boundary conditions.
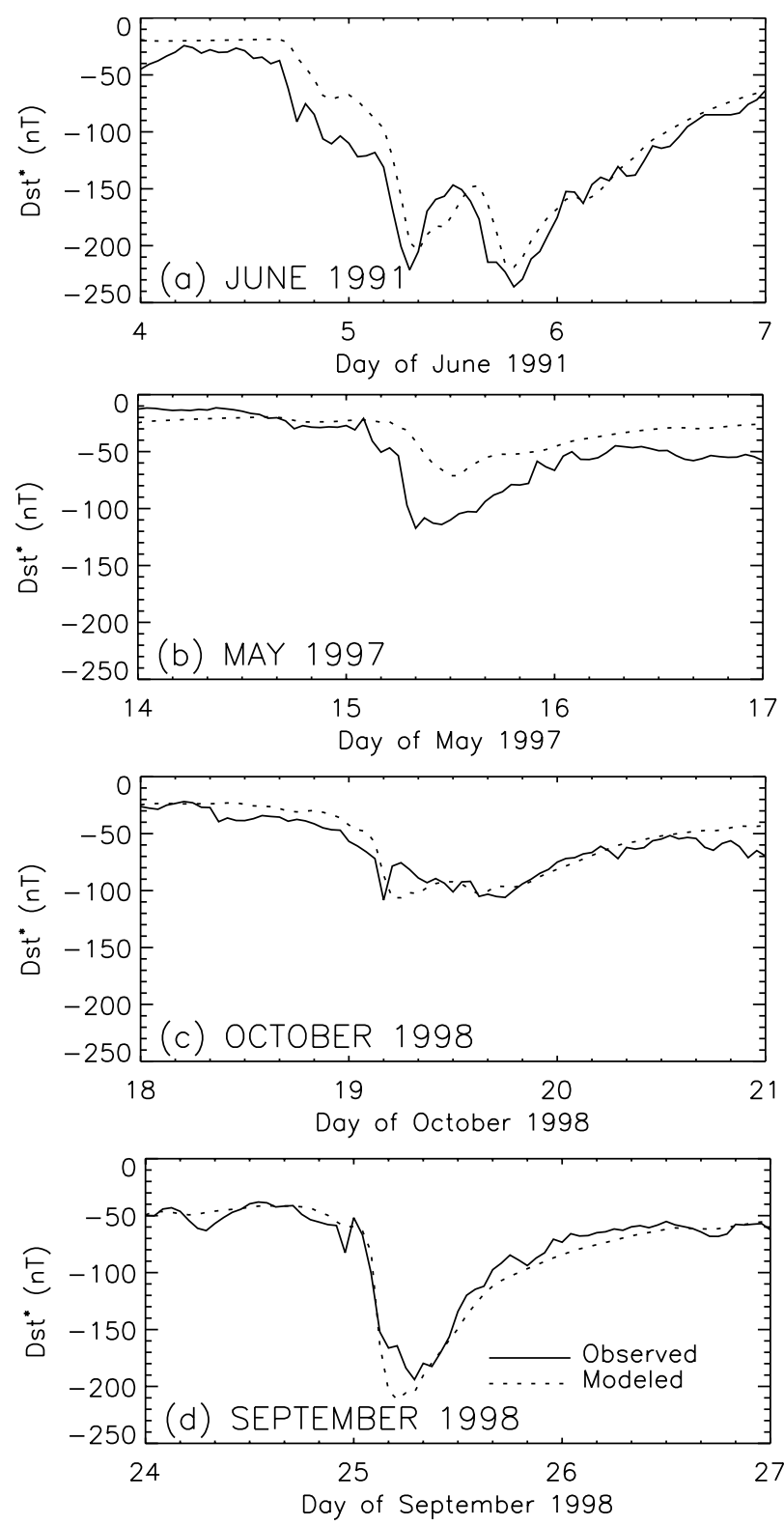

Figure 1. Observed (solid curves) and modeled (dotted curves) $D s t^{*}$ values for the four storms included in the analysis: (a) 5 June 1991, (b) 15 May 1997, (c) 19 October 1998, and (d) 25 September 1998.

[7] For this analysis, simulation results for four magnetic storms will be examined: 5 June 1991, 15 May 1997, 19 October 1998, and 25 September 1998. All four storms were driven by interplanetary coronal mass ejections (ICMEs), but the specific solar wind conditions were quite different between the events. Kozyra et al. [2002] (for the June 1991 storm) and Liemohn et al. [2001] (for the others) showed that the model accurately reproduced the observed features of the ion distributions. Figure 1 is a reproduction of the theory-data comparison of $D s t^{*}$ during the storm intervals. The observed $D s t^{*}$ values are calculated from the Kyoto hourly Dst index by correcting for magnetopause currents and the diamagnetic influence of Earth [Kozyra et al., 1998], while the model results use the Dessler-Parker-Sckopke (DPS) relation to equate the globally averaged magnetic perturbation to the total energy of the ring current [Dessler and Parker, 1959; Sckopke, 1966; Carovillano and Siscoe, 1973]. Figure 1 shows that Dst* is accurately reproduced for three of the four storms, with an under- 
estimation in the intensity of the May 1997 storm. The shape of the $D s t^{*}$ profile for this storm is similar between the observed and modeled values, so the magnitude difference does not invalidate the simulation results. In section 3 the ion source and loss rate results from these storm time ring current calculations will be examined to determine the significance of adiabatic drift-induced energization in the energy budget of the inner magnetosphere.

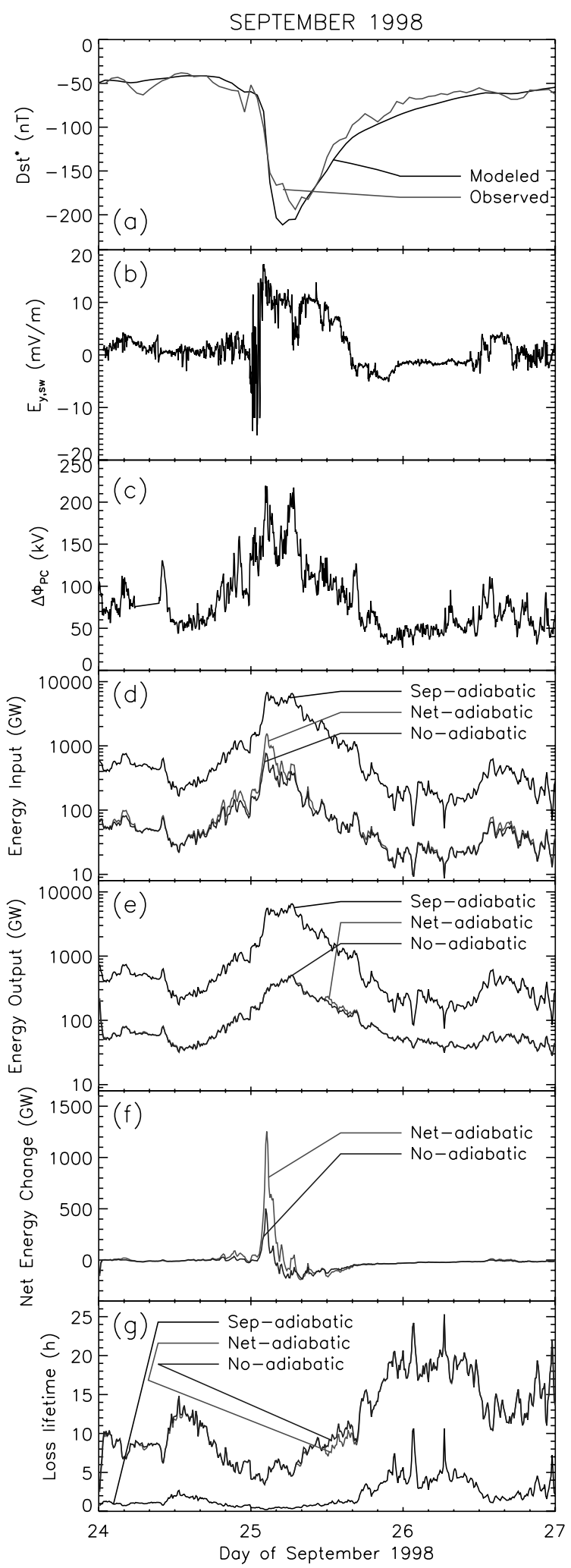

\section{Results}

[8] For conciseness, only the 25 September 1998 storm is considered in detail. The other storms exhibit a very similar trend seen during this event. Results from all four storms are then included in a comparison of the energy input and output rates versus the solar wind motional electric field $E_{y, \text { sw }}$. Results from all of the storms are also used in a final examination of the influence of the chosen convection electric field description in the inner magnetosphere.

\subsection{Case Study: The September 1998 Storm}

[9] Results for the interval of 24-26 September 1998 are presented in Figure 2. Figure 2a shows the Dst* values (modeled and observed) again for timing reference. This storm has a rapid drop in $D s t^{*}$ early on the 25 th followed by a classic two-phase decay signature. The early recovery phase lasted roughly 12 hours, with the late recovery phase starting in the final hours of the 25th and extending throughout the next day. The storm was large, with an observed $D s t^{*}$ minimum of $-194 \mathrm{nT}$.

[10] One of the essential drivers of inner magnetospheric dynamics is the convection electric field, which has been closely related to $E_{y, \mathrm{sw}}$ [cf. Reiff et al., 1981; Weimer, 1995]. The $y$ component of the solar wind electric field is shown in Figure $2 b$. It is obtained simply by multiplying the bulk speed of the solar wind with the $z$ component of the interplanetary magnetic field (IMF). The ionospheric cross-polar cap potential difference $\Delta \Phi_{\mathrm{PC}}$ is shown in Figure 2c. It is calculated by deconvolving highlatitude magnetometer data with the assimilative mapping of ionospheric electrodynamics (AMIE) technique [Richmond and Kamide, 1988] and taking the maximum minus the minimum values of the resulting potential pattern. The IMF is southward for positive values of $E_{y, \mathrm{sw}}$, so these periods have strong coupling between the solar wind and magnetosphere/ionosphere system. This is reflected in the increase of $\Delta \Phi_{\mathrm{PC}}$ during such intervals. However, the coupling is not one-to-one, as other processes also control the ionospheric potential drop (such as ionospheric conductivity and field-aligned current intensity). There appears to be two roughly equal intervals of strong $E_{y, \mathrm{sw}}$, the first associated with the sheath and the second with the magnetic cloud. The main phase only occurs during the first interval, while the recovery occurs during the passage of the second interval. Dst* increases are not expected during intervals of large positive $E_{y, \mathrm{sw}}$ [cf. Burton et al., 1975; Akasofu, 1981; O'Brien and McPherron, 2000]. For this storm the second interval of strong $E_{y, \mathrm{sw}}$ was ineffective because of a major substorm that was triggered by the slight northward turning of the IMF at the sheath-cloud interface (J. U. Kozyra et al., manuscript in preparation, 2001). This substorm essentially emptied the tail, and although convection remained strong throughout the cloud passage, there was no plasma to move into the near-Earth region. Therefore $D s t^{*}$ recovered during this period as the highdensity plasma from the sheath passage was replaced by lowdensity plasma.

Figure 2. (opposite) Time series of various ring current quantities during the September 1998 magnetic storm. Shown are (a) modeled and observed $D s t^{*}$, (b) solar wind motional electric field, (c) ionospheric cross-polar cap potential difference, (d) energy input rate, (e) energy output rate, (f) net energy change rate, and $(\mathrm{g})$ loss lifetime. Figures $2 \mathrm{~d}-2 \mathrm{~g}$ show a comparison of these quantities with the adiabatic drift energization and deenergization included separately in the input and output rates, respectively (black curve labeled "sep-adiabatic"), with only the net influence included in either the source or the loss term, as appropriate (red curve labeled "net-adiabatic"), and without the adiabatic influence included (blue curve labeled "no-adiabatic"). See color version of this figure at back of this issue. 
[11] Figures $2 d-2 g$ show energy gain and loss rates for this time interval. There are two sources of ion energy in the calculations, inflow through the outer boundary of the simulation domain and adiabatic energization inside of the domain. All of the energy input rate values include the nightside particle inflow. The difference between the three energy input curves in Figure 2d is the inclusion of adiabatic energization (black line labeled "sepadiabatic"), the inclusion of the net-adiabatic energization (gain minus loss) only when this quantity is positive (red line labeled "net-adiabatic"), and the omission of adiabatic energization from this summation (blue line labeled "no-adiabatic").

[12] There are several loss mechanisms of ion energy in the calculations. The energy losses from dayside particle outflow through the simulation boundary, precipitation into the atmosphere, charge exchange with the geocorona, and energy decay from Coulomb collisions with the thermal plasma are included in all three of the energy output curves in Figure 2e. The difference between the three curves is with the inclusion of all of the adiabatic deenergization inside the simulation domain (black line, sepadiabatic), the net-adiabatic energization (gain minus loss) only when this quantity is negative (red line, net-adiabatic), and the omission of adiabatic deenergization from this summation (blue line, no-adiabatic).

[13] The entire energy range of the simulation is included in these calculations $(10 \mathrm{eV}$ to $400 \mathrm{keV})$, and it is assumed that this range covers all of the ring current ion energy. Because $D s t^{*}$ has been shown to be directly proportional to total energy of the ring current (through the DPS relation), energy input and output rates are chosen for this display. Particle flow rates are susceptible to bias due the energy-dependent nature of the ring current source and loss mechanisms. Energy change rates, however, should reflect the true proportion of changes in ring current strength due to the processes considered.

[14] It is seen that in Figures 2d and 2e (energy input and output, respectively), the net-adiabatic curve is just slightly higher than the no-adiabatic curve, while the sep-adiabatic curve is nearly an order of magnitude higher than these values. This is true not only during the storm but also before and after it, although the total input and output rates are an order of magnitude lower at these quiet times compared with the peak of the disturbance. The quiet time adiabatic drift values are from the small oscillations in $L$ shell in the ions' trajectories around Earth. These plots show that the constant energization and deenergization is actually much greater than the loss rate of the symmetric ring current. The energy input and output rates are very well correlated with $\Delta \Phi_{\mathrm{PC}}$ (Figure 2c) and also with $D s t^{*}$ (Figure 2a) and $E_{y, \mathrm{sw}}$ (Figure 2b). The correlation with $\Delta \Phi_{\mathrm{PC}}$ is expected because this is the driver used in the convection electric field formulation in the simulation runs. The correlation with $E_{y, \mathrm{sw}}$ is expected because of this electric field is the energy source for the ionospheric electric potential. The correlation with $D s t *$ is expected because more ring current energy means more particles drifting through the inner magnetosphere.

[15] Figure $2 \mathrm{f}$ shows the total energy rate of change (input minus output from Figures $2 \mathrm{~d}$ and $2 \mathrm{e}$ ) during the storm interval. It is seen that the energy gain from adiabatic drift is larger than the loss (note that there is no sep-adiabatic curve for this plot). During the main phase of the storm (compare timing with Figure 2a), the net energy gain from adiabatic drift provides $60 \%$ of the energy gain responsible for the growth of the ring current (difference between the red and blue curves). This is similar to the finding of Lyons and Williams [1980], who claimed that the storm time ring current buildup inside of $4 R_{E}$ could be accounted for by adiabatic energization. However, instead of this buildup simply the being inward displacement of the preexisting symmetric ring current from farther out, the ions are actually flowing through the inner magnetosphere [cf. Liemohn et al., 2001; Kozyra et al., 2002]. Therefore it is more similar to the findings of Lee et al. [1982,
1983] and Chen et al. [1993, 1994], who found that adiabatic energization was the dominant source term for the low-energy ring current ions. Chen et al. [1994] found storm time ion phase space density enhancements for $E \sim 30-160 \mathrm{keV}$ for $L \sim 2.5-4$ primarily caused by convective access of the near-Earth plasma sheet to the inner magnetosphere. Higher-energy ions $(E>160$ $\mathrm{keV}$ ) are injected into this region only during long-duration storms ( $>6$ hour main phase) through radial diffusion. This is very similar to the results of the present simulations, in which the near-Earth ring current is dominated by ions of $E<160 \mathrm{keV}$ [cf. Liemohn et al., 2001; Kozyra et al., 2002]. In the present simulations, radial diffusion is only included through the stochastic variations in the electric field, which can cause drift shell migration for the higherenergy ions. This is, in fact, the source of the diffusion parameterized by the radial diffusion coefficient $D_{\mathrm{LL}}$ in the Chen et al. [1993, 1994] simulations, although they used a larger amplitude variation (on the order of the mean field) in the calculation of $D_{\mathrm{LL}}$.

[16] During the recovery phase the influence of adiabatic drift on the net energy gain is still almost always positive. During the late recovery phase, the influence of adiabatic cross- $L$ drift is essentially zero. The preference toward gain is because the losses within the inner magnetosphere attenuate the ring current strength as the particles move from the nightside to the dayside, making adiabatic gains larger than adiabatic losses. That is, the energization experienced on the nightside by the inwardly drifting particles is not balanced by a comparable loss on the dayside. Two mechanisms are responsible for the energy loss: (1) Particles are lost in the inner magnetosphere and never make it to (or through) the dayside region (via charge exchange or atmospheric precipitation), and (2) energy decay from Coulomb collisions decreases the kinetic energy of any ion that makes it completely through the inner magnetosphere. The former is by far the more significant effect, with charge exchange accounting for most of this loss [cf. Jordanova et al., 1998; Liemohn et al., 1999].

[17] Figure $2 \mathrm{~g}$ shows $e$-folding lifetimes of $D s t^{*}$, found by dividing the modeled $D s t^{*}$ profile in Figure 2a by the energy output profiles in Figure 2e. That is, if there were no further energy input, then $D s t^{*}$ would exponentially decay back toward zero with one of these timescales. It is seen that the loss lifetime is usually below 5 hours (even below 1 hour near the $D s t^{*}$ minimum) when the adiabatic losses on the dayside are included in the definition of energy output. With only the net influence (when the total adiabatic drift energization is negative) or without adiabatic effects included in this definition, the loss lifetime reaches 5 hours only during the peak of the storm and is otherwise between 10 and 25 hours. As seen Figure 2e, it clear that the dayside deenergization of the hot ions is the dominant loss term throughout this interval, but this loss term is completely (or at least mostly) canceled by adiabatic energization on the nightside.

[18] Figure $2 \mathrm{~g}$ shows an interesting phenomenon not seen in the earlier plots. The loss lifetime before the storm without adiabatic effects included is less than 10 hours, substantially less than the lifetime during the late recovery phase of the storm. This is because the symmetric ring current is relatively weak at this time, consisting mainly of high-energy protons that have a very long charge exchange loss lifetime (many days). This allows the flow of plasma sheet ions farther out to dominate the energy output rate and thus the loss lifetime. The prestorm convection is driven by a $\Delta \Phi_{\mathrm{PC}}$ averaging $70 \mathrm{kV}$, which is strong enough to drive some ions through the inner magnetosphere. The main source and loss at this time is actually flow through the simulation boundaries rather than decay of the ion distribution in the inner magnetosphere. The timescale of this flow-through is partially reflected in this total loss lifetime, but the flow-through timescale is in fact faster than the total loss lifetime because of the presence of a 50-nT preexisting ring current. However, the calculated loss timescale shown in 


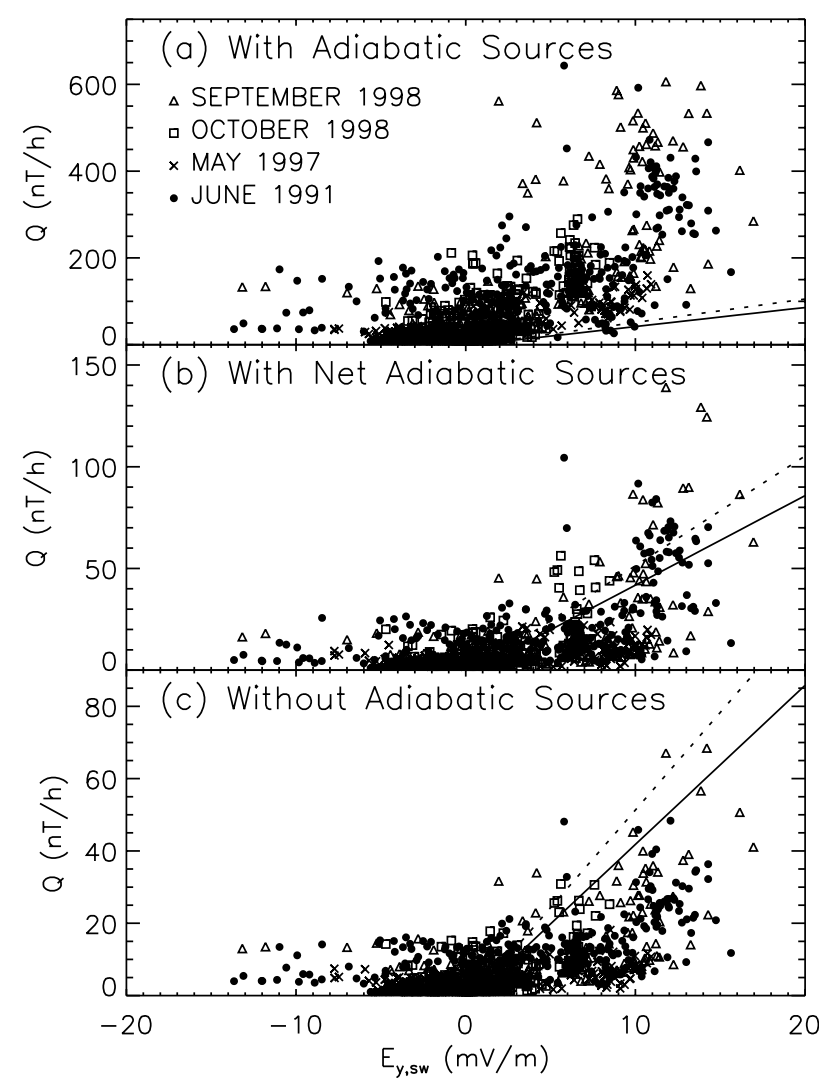

Figure 3. Ring current energy source rate versus solar wind electric field for (a) adiabatic gains included, (b) net-adiabatic gain included (when positive), and (c) no-adiabatic gains included. The solid and dotted curves are the source term functions for the O'Brien and McPherron [2000] and the Burton et al. [1975] predictor methods, respectively. The symbols are 10-min averages of simulation results for the four storms as indicated.

Figure $2 \mathrm{~g}$ does not translate into a $D s t^{*}$ decay rate because of a nearly identical inflow rate through the nightside boundary.

\subsection{Relation to Solar Wind Electric Field}

[19] As stated earlier, the driver of this adiabatic drift influence is the convection electric field. Because this field is closely related to $E_{y, \mathrm{sw}}$, it is useful to compare these source and loss rates with this quantity. While AMIE-derived $\Delta \Phi_{\mathrm{PC}}$ values are available for the four storms being examined, $E_{y, \mathrm{sw}}$ will be used for this comparison because of its use in previous studies that quantify ring current energization [cf. Burton et al., 1975; Akasofu, 1981; O'Brien and McPherron, 2000]. Figure 3 shows this comparison for the energy input rate for all four storms (each symbol represents a 10-min average during a 3-day interval encompassing the storm). The three panels correspond to the three curves in Figure 2d. Also shown in each panel are the source functions from the Burton et al. [1975] and O'Brien and McPherron [2000] predictor methods. These two were chosen because they have simple relationships between $E_{y, \text { sw }}$ and their energy source and loss rates. While there is significant scatter in the model results, a few conclusions can be drawn from them. It is seen in Figure 3 that the simulation results that include the net-adiabatic sources (when positive) best match the empirically derived source functions of the Dst predictor methods. The simulation result values are noticeably higher than the predictor method values when all of the adiabatic energy source is included in the definition of $Q$, and they are noticeably lower when none of this energy source is counted. Another feature of Figure 3 is that there is no systematic difference in the values between the storms, supporting the premise that the results are true for ICME-driven storms in general (and perhaps even more broadly applicable). Note that because only four storms are being considered, curve fits through the simulation results have not been calculated.

[20] A similar scatterplot for the loss lifetimes as a function of $E_{y, \text { sw }}$ is shown in Figure 4 (again, each symbol is a 10 -min average). The empirically derived t functions from the Burton et al. [1975] and O'Brien and McPherron [2000] predictor methods are shown for comparison. As seen in Figure 4a (values which include the dayside adiabatic deenergization term), all of the storms have many loss lifetime values of less than 1 hour for both positive and negative $E_{y, \mathrm{sw}}$. These small $\tau$ values at large negative $E_{y, \mathrm{sw}}$ appear during the recovery phase of the storms when the IMF swings northward. The total energy of the ring current is still substantial, and so the trickle of dayside outflow, combined with the small $L$ shell oscillation of the trapped ions, gives rise to a very small loss lifetime.

[21] As in Figure 3, the timescales calculated with the netadiabatic influence (when negative) has the best fit to the empirical values. However, because of the smallness of the net-adiabatic loss compared with the other loss processes (for reasons stated in section 3.1), Figures $4 \mathrm{~b}$ and $4 \mathrm{c}$ are very similar.

[22] In addition, as with the source rates in Figure 3, the $\tau$ values are similar for all four storms. The only storm with a slightly different trend is the May 1997 storm, with a number of points near $E_{y, \mathrm{sw}}=0$ with very large $\mathrm{t}$ values (reaching 25 hours in Figure $4 \mathrm{a}$ and exceeding 80 hours in Figures $4 \mathrm{~b}$ and $4 \mathrm{c}$ ). These points occur before the storm commencement when both the symmetric ring current and the gradually flowing partial ring

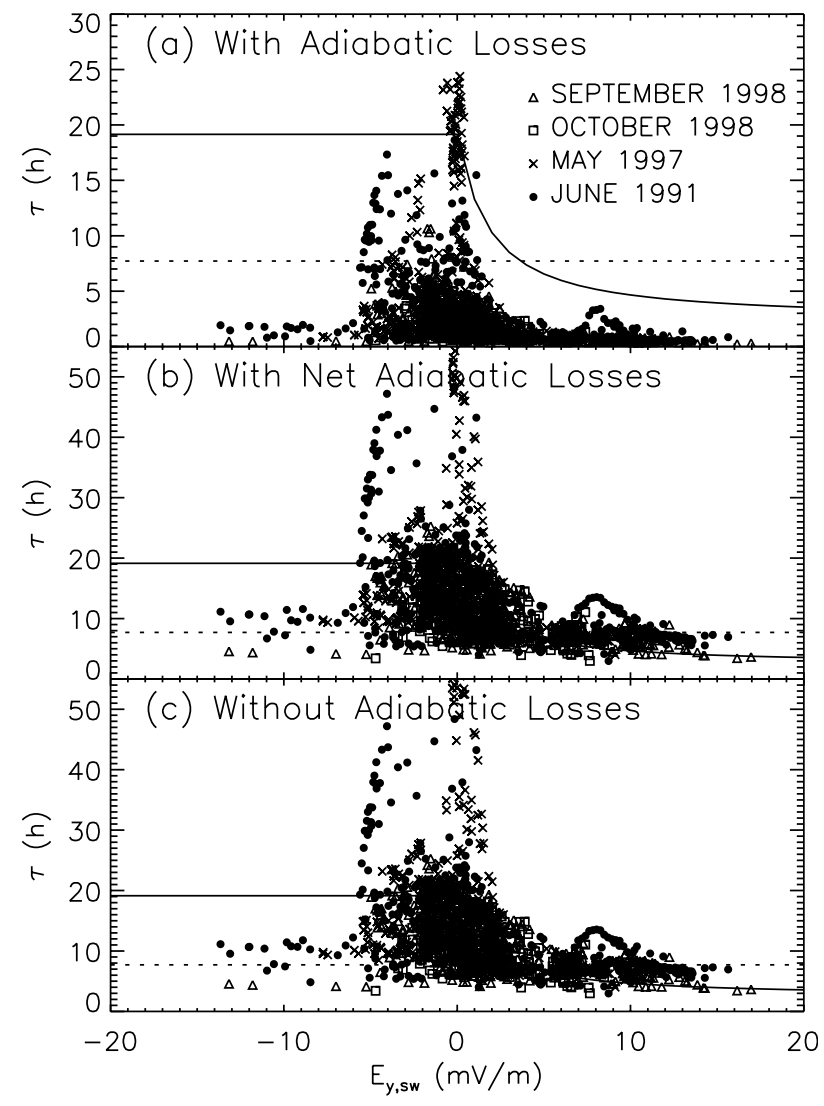

Figure 4. Like Figure 3 except for the loss lifetime instead of the source rate. 


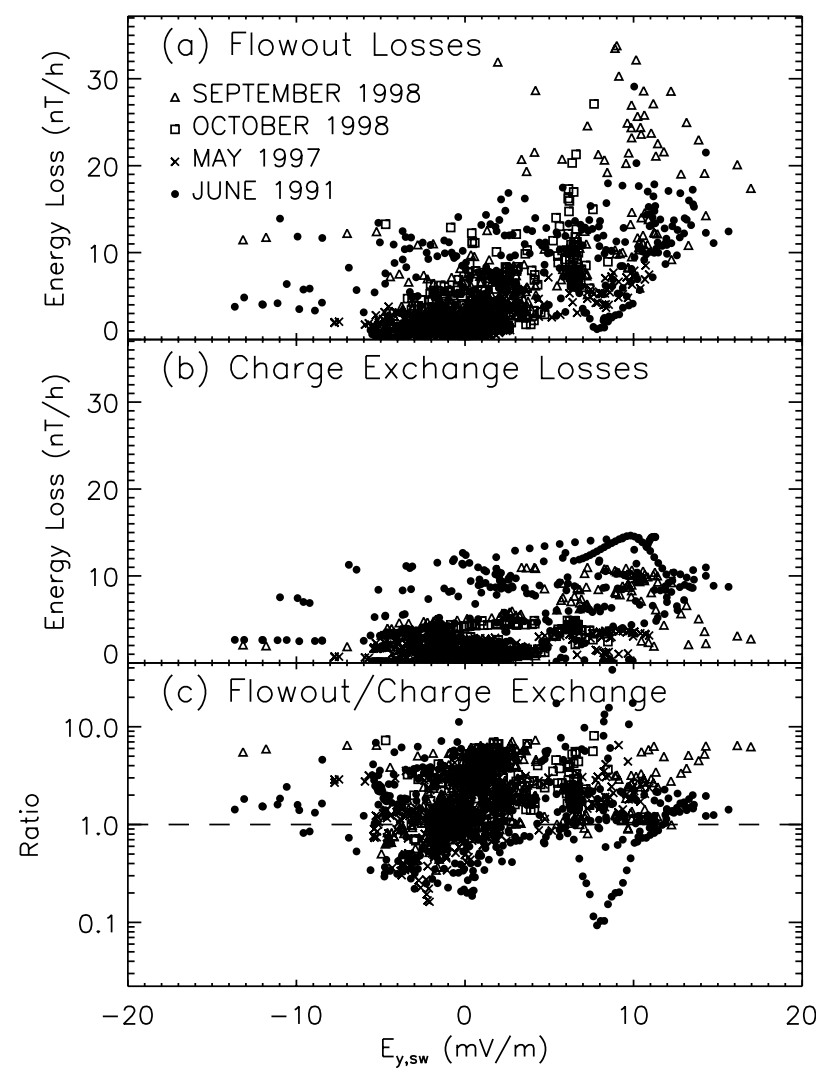

Figure 5. Nonadiabatic losses versus solar wind electric field for the four storms: (a) energy loss from dayside flow out, (b) energy loss from charge exchange collisions, and (c) the ratio of the two. In Figure $5 \mathrm{c}$ a dashed line is drawn at unity for reference.

current are very weak. These timescales reflect the high-energy $\mathrm{H}^{+}$ charge exchange decay lifetime, the dominant species, and loss process during this time interval.

[23] A question to be addressed in relation to Figure 4 is what physical processes are causing the smaller loss lifetimes at higher $E_{y, \mathrm{sw}}$. The difference between Figures $4 \mathrm{a}$ and $4 \mathrm{~b}$ indicates that adiabatic deenergization is a dominant loss mechanism regardless of $E_{y, \mathrm{sw}}$, but there is certainly a trend in Figures $4 \mathrm{~b}$ and $4 \mathrm{c}$ that suggest a correlation between high $E_{y, \mathrm{sw}}$ and small loss lifetime, as was found empirically by O'Brien and McPherron [2000]. As a physical interpretation of their findings, O'Brien and McPherron [2000] explained this $E_{y, \text { sw }}$ control of loss as a result of enhanced charge exchange due to the compression of the ring current during periods of enhanced $E_{y, \text { sw }}$. However, they assumed the ring current only exists on closed drift paths. Because it is largely on open drift paths during magnetic storms (a partial ring current), flow of ions out the dayside magnetopause can also be a major loss process. The physical description behind their functional form for the loss lifetime dependence on $E_{y, \mathrm{sw}}$ (using the trapping boundary location as a connection between loss and $\left.E_{y, \text { sw }}\right)$ is still correct, however, because flow-out intensity is also directly proportional to the trapping boundary. In fact, flow out should be even more correlated with $E_{y, \mathrm{sw}}$ than charge exchange, because a sudden drop in $E_{y, \text { sw }}$ will instantaneously cut off flow out, but the compressed ring current will not immediately expand (that is, there will be a period of high charge exchange loss during low $E_{y, \mathrm{sw}}$ values).

[24] The modeling results of the present study can elucidate the issue of which loss process (flow out or charge exchange) is dominant during high $E_{y, \text { sw }}$. Figure 5 shows the magnitude of the flow-out energy losses (Figure 5a) and charge exchange energy losses (Figure $5 \mathrm{~b}$ ) to $E_{y, \mathrm{sw}}$ for the four storms. It can be clearly seen that at large $E_{y, \mathrm{sw}}$ values, flow out is the dominant loss process of these two. It can also be seen, however, that flow out is equal to or greater than charge exchange when $E_{y, \mathrm{sw}}$ is near zero or even negative. This is a peculiar result, as one would expect charge exchange to dominate at these slow-convection times.

[25] The reason for flow-out dominance for $E_{y, \mathrm{sw}} \leq 0$ has already been mentioned in the discussion of Figure $2 \mathrm{~g}$. That is, the convection strength does not go to zero when $E_{y, \mathrm{sw}}$ goes to zero, and therefore open drift paths through the inner magnetosphere exist at all times. This convection is a significant source and loss of hot ions compared with the slow charge exchange process. To elucidate this comparison, Figure $5 \mathrm{c}$ shows the ratio of the energy loss rate from flow out to that from charge exchange as a function of $E_{y, \mathrm{sw}}$ for the four storms. Qualitatively, it appears the ratio is usually $>1$, especially for positive $E_{y, \mathrm{sw}}$. Quantitatively, the average ratio is 2.2 for all $E_{y, \mathrm{sw}}$ values, 2.6 for $E_{y, \mathrm{sw}}>0$, and 1.6 for $E_{y, \text { sw }}<0$. That this ratio averages to $<1$ even for negative solar wind electric field values indicates that dayside flow out is a bigger loss process for inner magnetosphere hot-ion energy than charge exchange, even before storms and during their late recovery phases. However, this does not translate directly into a flow-outloss control of the decay rate of $D s t^{*}$, because there is also a source of ion energy during such times. Figure $3 \mathrm{c}$ shows energy source rates from 0 to $16 \mathrm{nT} / \mathrm{h}$ for near-zero or negative $E_{y, \mathrm{sw}}$, and Figure $3 \mathrm{~b}$ (net adiabatic energization included) ranges from 0 to $25 \mathrm{nT} / \mathrm{h}$ energy input. This is comparable to the energy loss rates from flow out $(0-14 \mathrm{nT} / \mathrm{h})$ and charge exchange $(0-12 \mathrm{nT} / \mathrm{h})$ for this range. Without doing a full trajectory analysis for all of phase space during all of the low $E_{y, \mathrm{sw}}$ times, it is difficult to determine which of these loss processes actually accounts for the loss lifetime of $D s t^{*}$ during such times. Even though the convection electric field is small when $E_{y, \mathrm{sw}}$ is negative, its chaotic variability (along with the differential drift periods across the ring current ion energy range) results in ions being continually captured onto trapped drift paths and lost onto open drift paths. Thus there is no clear one-toone relationship between the ions injected into the inner magneto-

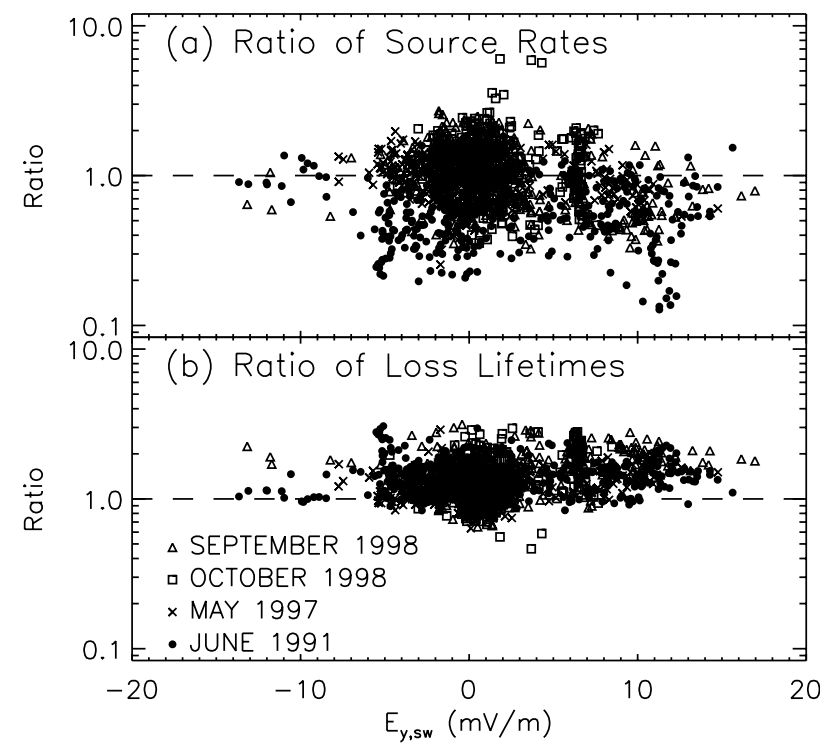

Figure 6. Ratios of the (a) source rate and (b) loss lifetime from the simulations using the modified McIlwain [1986] electric field to those using the Volland-Stern electric field for the four storms. A dashed line is drawn at unity for reference. 

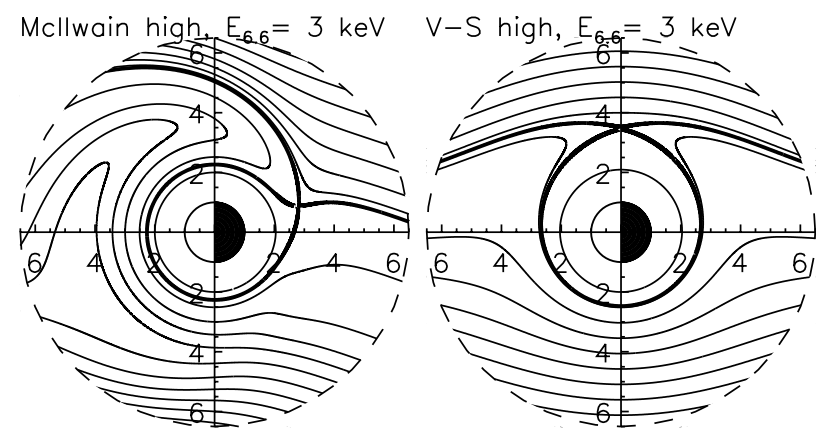

Figure 7. Drift trajectory patterns in the inner magnetosphere for ions with $3 \mathrm{keV}$ at geosynchronous orbit $(0.0278 \mathrm{keV} / \mathrm{nT})$ for typical high-activity parameters for (a) the modified McIlwain field and (b) the Volland-Stern field.

sphere on the nightside and through which loss process such ions will eventually exit near-Earth space.

\subsection{Dependence on Convection Electric Field Description}

[26] All of the results so far have shown the source and loss rates using a modified McIlwain [1986] convection electric field. Because of the strong influence of this field description on the dynamics of the storm time ring current, it is useful to compare these results against those using a different convection model. Figure 6 shows such a comparison, again for all 4 storms, showing the ratio of the results using the modified Mcllwain field to those using the Volland-Stern field. Liemohn et al. [2001] gives a description of the similarities and differences between these electric field models. The quantities used in these plots are the net source rates and loss lifetimes including the net-adiabatic changes. While there is considerable spread in the source rate ratio (Figure 6a), the average value is 1.01 , extremely close to unity. This means the two field descriptions deliver roughly the same amount of energy to the inner magnetosphere. However, for considering only those values with $E_{y, \mathrm{sw}} \geq 5 \mathrm{mV} / \mathrm{m}$, this average value drops to 0.84 . This difference results from the fact that neither field description has a simple strength dependence on $E_{y, \mathrm{sw}}$ and that the Volland-Stern model has a slightly higher convection strength than the modified McIlwain field at these times.

[27] The loss rate ratio (Figure 6b) shows a skew in the ratio to values greater than unity for all $E_{y, \text { sw }}$ values, with an average ratio of 1.39. This means that the Volland-Stern model has systematically smaller loss lifetimes, regardless of convection strength. This is a result of the morphology of the flow pattern of hot ions in the inner magnetosphere. Figure 7 shows ion drift paths for typical high activity values for these convection descriptions. The trajectories are drawn for particles with $3 \mathrm{keV}$ of energy at geosynchronous orbit, which is near (a bit below) the average ion temperature at this altitude. It can be seen that the modified McIlwain field wraps the trajectories around the dayside magnetosphere more than the Volland-Stern field does. This dependence on the flow pattern of hot ions through the inner magnetosphere makes the choice of the convection electric field a critical element in ring current modeling.

[28] Korth et al. [2002] recently demonstrated the lack of predictability for the inner magnetospheric electric field. In attempting to tomographically invert the hydrogen geocoronal densities from compilations of MPA data, they discovered that none of the electric field models (including analytical and empirical descriptions) could produce a realistic density distribution inside of $L \sim 4$. While many factors contribute to this inability to perform a viable inversion, discrepancies between the electric field descriptions and the true electric field contribute (at least to some degree) to the error. In spite of the findings of Korth et al. [2002], the model used in the present study reproduces the observations of the events. Therefore the results presented in section 3 are thought to be an accurate representation of the storm time ring current strength and its growth and decay via various processes.

\section{Conclusions}

[29] It was shown that the adiabatic energy gain and loss due to drift across $L$-shell is large compared with other source and loss processes, even during geomagnetically very quiet times. During active periods the open drift path configuration of the hotion motion dominates the physics of the ring current, and thus it is not surprising that adiabatic drift across $L$ shells significantly contributes to the total energy changes. During these times this effect dominates because of the small oscillations in $L$ (caused by the convection electric field) in the ions drift motion around Earth. When energization and deenergization are counted separately as gain and loss terms, they dominate all other processes combined. However, they often balance each other, and so the net-adiabatic drift influence is usually not the dominant energy change process. The net influence of the adiabatic energy changes is skewed heavily toward energy gains, because some particles are lost in the inner magnetosphere before they adiabatically deenergize. Only during portions of the main phase of storms does this adiabatic energization dominate other processes, accounting for more than half of the total energization of the ring current but having a negligible contribution to the ring current decay. The source rates and loss lifetimes with only the net-adiabatic influence included (in one or the other, as appropriate) produce the best fit with the corresponding functions found by several Dst predictor methods. In addition, it was shown that the prestorm loss lifetime could be quite low or very high, depending on the strengths of the existing symmetric and partial ring currents.

[30] Inclusion of the adiabatic effects yields source and loss rates significantly different from the observationally based functions of the Dst predictor methods (Figures 3a and 4a). One explanation for this discrepancy is that the adiabatic influence is fast, less than an hour timescale, and the resolution of the Dst index is 1 hour, so it is hard to isolate this as an influence. Moreover, the empirically derived source and loss values are obtained in order to best reproduce the Dst index. They are not trying to resolve each physical mechanism and term that contributes to these values, merely the overall rates according to their data analysis method. The conclusion is that for consistent comparison between the source and loss rates from Dst predictor methods and those calculated from kinetic transport model results, one should include only the net-adiabatic drift influence (in addition to other source and loss effects).

[31] It was also shown that energy loss due to flow out is larger than that due to charge exchange. This is true especially for large positive values of $E_{y, \text { sw }}$, but it is also true for small and even negative $E_{y, \mathrm{sw}}$ values. However, this cannot be directly related to a statement about which of these processes dominates the empirically derived decay timescales for these $E_{y, \text { sw }}$ values [e.g., O'Brien and McPherron, 2000], because such timescales are calculated assuming no energy input below some threshold $E_{y, \text { sw }}$ value (typically $+0.5 \mathrm{mV} / \mathrm{m}$ ). As shown in these computational results of the storm time ring current, for negative values of $E_{y, \mathrm{sw}}$, there is (on average) a nonzero energy source and a loss lifetime smaller than that assumed in Dst predictor methods. This is because the primary driver of the convection pattern used in the ring current simulations, $\Delta \Phi_{\mathrm{PC}}$, does not go to zero when $E_{y, \mathrm{sw}}$ goes to zero. Therefore open drift paths exist at all times, and flow out can be (and often is) larger than charge exchange losses in the inner magnetosphere. The exact specification of when this occurs, however, depends on the relative strengths of the preexisting ring current and near-Earth plasma sheet. Resolution of this issue 
regarding Dst prediction when $E_{y, \mathrm{sw}}$ is negative requires further examination.

[32] Acknowledgments. This work was supported by NASA grants NAG5-4771, NAG5-10297, and NAG-10850, and NSF contracts ATM9711381, ATM-9800830, ATM-9801941, and ATM-0090165. The authors would also like to thank all of the data providers that make the ring current simulations possible, especially the Wind and ACE particle and field instrument teams and CDAWeb for access to this data, the Air Force Research Laboratory at Hanscom AFB, and the Kyoto World Data Center.

[33] Janet G. Luhmann thanks Margaret W. Chen and Richard M. Thorne for their assistance in evaluating this paper.

\section{References}

Akasofu, S.-I., Energy coupling between the solar wind and the magnetosphere, Space Sci. Rev., 28, 121, 1981.

Bame, S. J., et al., Magnetospheric plasma analyzer for spacecraft with constrained resources, Rev. Sci. Instrum., 64, 1026, 1993.

Belian, R. D., G. R. Gisler, T. Cayton, and R. Christensen, High Z energetic particles at geosynchronous orbit during the great proton event of October 1989, J. Geophys. Res. 97, 16,897, 1992.

Birn, J., M. F. Thomsen, J. E. Borovsky, G. D. Reeves, D. J. McComas, and R. D. Belian, Characteristic plasma properties during dispersionless substorm injections at geosyncronous orbit, J. Geophys. Res., 102, 2309, 1997.

Burch, J. L., et al., Views of Earth's magnetosphere with the IMAGE satellite, Science, 291, 619, 2001.

Burton, R. K., R. L. McPherron, and C. T. Russell, An empirical relationship between interplanetary conditions and Dst, J. Geophys. Res., 80, 4204, 1975.

Carovillano, R. L., and G. L. Siscoe, Energy and momentum theorems in magnetospheric processes, Rev. Geophys., 11, 289, 1973.

Chen, M. W., M. Schulz, L. R. Lyons, and D. J. Gorney, Stormtime transport of ring current and radiation belt ions, J. Geophys. Res., 98, $3835,1993$.

Chen, M. W., L. R. Lyons, and M. Schulz, Simulations of phase space distributions of storm time proton ring current, J. Geophys. Res., 99, $5745,1994$.

DeForest, S. E., and C. E. McIlwain, Plasma clouds in the magnetosphere, J. Geophys. Res., 76, 3587, 1971.

Dessler, A. J., and E. N. Parker, Hydromagnetic theory of geomagnetic storms, J. Geophys. Res., 64, 2239, 1959.

Ebihara, Y., and M. Ejiri, Simulation study on fundamental properties of the storm-time ring current, J. Geophys. Res., 105, 15,843, 2000.

Fok, M.-C., J. U. Kozyra, A. F. Nagy, C. E. Rasmussen, and G. V. Khazanov, A decay model of equatorial ring current and the associated aeronomical consequences, J. Geophys. Res., 98, 19,381, 1993.

Gonzalez, W. D., J. A. Joselyn, Y. Kamide, H. W. Kroehl, G. Rostoker, B. T. Tsurutani, and V. M. Vasyliunas, What is a geomagnetic storm? J. Geophys. Res., 99, 5771, 1994.

Greenspan, M. E., and D. C. Hamilton, A test of the Dessler-ParkerSckopke relation during magnetic storms, J. Geophys. Res., 105, 5419, 2000 .

Jordanova, V. K., L. M. Kistler, J. U. Kozyra, G. V. Khazanov, and A. F. Nagy, Collisional losses of ring current ions, J. Geophys. Res., 101, 111, 1996.

Jordanova, V. K., C. J. Farrugia, L. Janoo, J. M. Quinn, R. B. Torbert, K. W. Ogilvie, R. P. Lepping, J. T. Steinberg, D. J. McComas, and R. D. Belian, October 1995 magnetic cloud and accompanying storm activity: Ring current evolution, J. Geophys. Res., 103, 79, 1998

Jordanova, V. K., R. B. Torbert, R. M. Thorne, H. L. Collin, J. L. Roeder, and J. C. Foster, Ring current activity during the early $\mathrm{Bz}<0$ phase of the January 1997 magnetic cloud, J. Geophys. Res., 104, 24,895-24,914, 1999.

Jorgensen, A. M., M. G. Henderson, E. C. Roelof, G. D. Reeves, and H. E.
Spence, Charge exchange contribution to the decay of the ring current, measured by energetic neutral atoms (ENAs), J. Geophys. Res., 106, 1931, 2001.

Korth, H., M. F. Thomsen, K.-H. Glassmeier, and W. S. Phillips, Particle tomography of the inner magnetosphere, J. Geophys. Res., 10.1029/ 2001JA000147, in press, 2002

Kozyra, J. U., V. K. Jordanova, J. E. Borovsky, M. F. Thomsen, D. J. Knipp, D. S. Evans, D. J. McComas, and T. E. Cayton, Effects of a high-density plasma sheet on ring current development during the November 2-6, 1993, magnetic storm, J. Geophys. Res., 103, 26,285, 1998. Kozyra, J. U., M. W. Liemohn, C. R. Clauer, A. J. Ridley, M. F. Thomsen, J. E. Borovsky, J. L. Roeder, and V. K. Jordanova, Multistep Dst development and ring current composition changes during the 4-6 June 1991 magnetic storm, J. Geophys. Res., 10.1029/2001JA000023, in press, 2002.

Lee, L. C., J. R. Kan, and S.-I. Akasofu, Ring current energy injection rate and solar wind-magnetosphere energy coupling, Planet. Space Sci., 30, 627, 1982.

Lee, L. C., G. Corrick, and S.-I. Akasofu, On the ring current energy injection rate, Planet. Space Sci., 31, 901, 1983.

Liemohn, M. W., J. U. Kozyra, V. K. Jordanova, G. V. Khazanov, M. F. Thomsen, and T. E. Cayton, Analysis of early phase ring current recovery mechanisms during geomagnetic storms, Geophys. Res. Lett., 25, 2845, 1999.

Liemohn, M. W., J. U. Kozyra, M. F. Thomsen, J. L. Roeder, G. Lu, J. E. Borovsky, and T. E. Cayton, The dominant role of the asymmetric ring current in producing the stormtime Dst*, J. Geophys. Res., 106, 10,883, 2001.

Lyons, L. R., and D. J. Williams, A source for the geomagnetic storm main phase ring current, J. Geophys. Res., 85, 523, 1980.

Mcllwain, C. E., A $K p$-dependent equatorial electric field model, $A d v$. Space Res., 6(3), 187, 1986.

Mitchell, D. G., K. C. Hsieh, C. C. Curtis, D. C. Hamilton, H. D. Voss, E. C. Roelof, and P. C. Brandt, Imaging two geomagnetic storms in energetic neutral atoms, Geophys. Res. Lett., 28, 1151, 2001.

O'Brien, T. P., and R. L. McPherron, An empirical phase-space analysis of ring current dynamics: Solar wind control of injection and decay, J. Geophys. Res., 105, 7707, 2000.

Pollock, C. J., et al., First medium energy neutral atom (MENA) images of Earth's magnetosphere during substorm and storm-time, Geophys. Res. Lett., 28, 1147, 2001

Reeves, G. D., and H. E. Spence, Charge exchange contribution to the decay of the ring current measured by energetic neutral atoms (ENAs), J. Geophys. Res., 106, 1931, 2001.

Reiff, P. H., R. W. Spiro, and T. W. Hill, Dependence of polar cap potential drop of interplanetary parameters, J. Geophys. Res., 86, 7639, 1981.

Richmond, A. D., and Y. Kamide, Mapping electrodynamic features of the high-latitude ionosphere from localized observations: Technique, J. Geophys. Res., 93, 5741, 1988.

Sckopke, N., A general relation between the energy of trapped particles and the disturbance field near the Earth, J. Geophys. Res., 71, 3125, 1966.

Weimer, D. R., Models of high-latitude electric potentials derived with a least errors fit to spherical harmonic coefficients, J. Geophys. Res., 100, 19,595, 1995.

C. R. Clauer, J. U. Kozyra, and M. W. Liemohn, Space Physics Research Laboratory, University of Michigan, 2455 Hayward St., Ann Arbor, MI 48109-2143, USA. (liemohn@umich.edu)

G. V. Khazanov, National Space Science and Technology Center, NASA Marshall Space Flight Center, 320 Sparkman Drive, Huntsville, AL 35805, USA. (george.khazanov@msfc.nasa.gov)

M. F. Thomsen, Atmospheric and Space Sciences, Los Alamos National Laboratory, P.O. Box 1663, Mail Stop D466, Los Alamos, NM 87545, USA. (mthomsen@lanl.gov) 


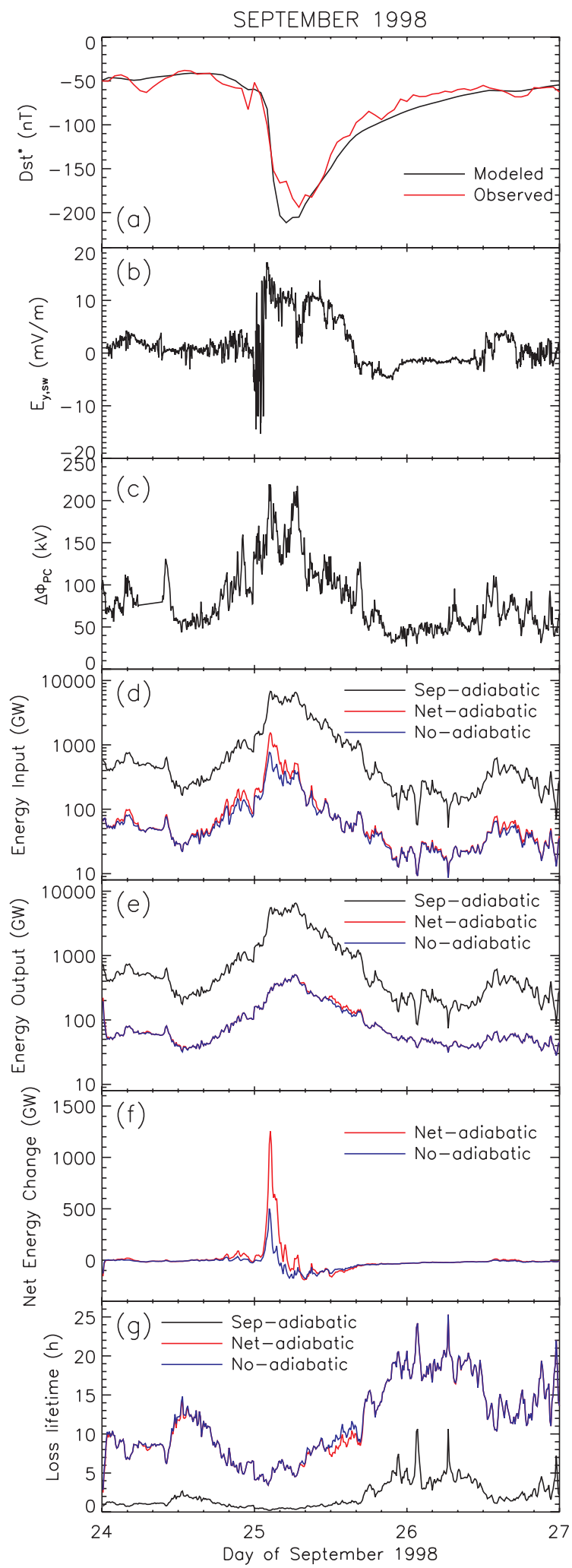

Figure 2. Time series of various ring current quantities during the September 1998 magnetic storm. Shown are (a) modeled and observed $D s t^{*}$, (b) solar wind motional electric field, (c) ionospheric cross-polar cap potential difference, (d) energy input rate, (e) energy output rate, (f) net energy change rate, and (g) loss lifetime. Figures $2 d-$ $2 \mathrm{~g}$ show a comparison of these quantities with the adiabatic drift energization and deenergization included separately in the input and output rates, respectively (black curve labeled "sep-adiabatic"), with only the net influence included in either the source or the loss term, as appropriate (red curve labeled "net-adiabatic"), and without the adiabatic influence included (blue curve labeled "no-adiabatic"). 